Background Neonatal septic shock is a devastating condition associated with high morbidity and mortality.

Methods A retrospective study was conducted in children's hospital Tunisian PICU between 2005 to 2013. All neonates $(<28$ days) treated for septic shock with bacterial proof were included. Nosocomial infection was an exclusion criteria. The chart review relieved demographics, length of stay, therapies and outcomes.

Results A total of 40 neonates were included. Mean age on admission was $34 \mathrm{~h} \pm 3.7$. Mean SNAPP score was $25 \pm 17$. Materno-fetal infection was observed in 37 cases, staphylococcal pneumonia in 2 cases and bacterial coinfection with bronchiolitis in the last case. The bacteriological study showed a notable predominance of streptococcus B (40\%) and E.coli (35\%). All patients required mechanical ventilation (mean duration: $85 \pm$ $556 \mathrm{~h}$ ) and haemodynamic support (mean duration $49 \pm 335$ h). Mortality rate was $19 \%$ in full term infants, $12.5 \%$ in near term infants and $27 \%$ in extremely preterm infants.

Conclusions Our results would indicate a high mortality rate in neonatal septic shock. A goal -directed therapy for septic shock, implanted in our PICU, could improve outcomes for this vulnerable population.

\section{PS-214 DOES A TOTAL STERILE COLLECTION BUNDLE REDUCE FALSE POSITIVE BLOOD CULTURE RATES AND ANTIBIOTIC USE IN NEONATAL INTENSIVE CARE?}

HE Gillett, LF Hamilton, JW Davis. Neonatal Intensive Care, St Michaels Hospital, Bristol, UK

10.1136/archdischild-2014-307384.513

Background and aim In neonatal intensive care coagulase negative Staphylococcus species can be both blood culture contaminant and pathogen. False positive cultures can result in clinical uncertainty and unnecessary antibiotic use. Our aim was to assess the effect of a total sterile blood culture collection bundle on the incidence of false positive blood cultures in a regional surgical neonatal intensive care unit.

Method Clinical data of all infants who had blood cultures taken before and after the introduction of the collection bundle (sterile technique and 2\% Chlorhexidine) were collected. The rates of false positive blood cultures, defined as the presence of a skin commensal and $<3$ predefined clinical signs (Modi et al. 2009), were compared.

Results In total 367 blood cultures from 294 babies were assessed, 197 pre-intervention (PRE) and 170 following bundle introduction (POST). The median birth weight and gestation were similar in both groups. The rate of false positive cultures in the total PRE group was $9 / 197(4.6 \%)$ compared to $1 / 170$ $(0.6 \%)$ in the POST group ( $<<0.05)$. In infants $<28$ weeks' the rates reduced from $4 / 29(13.8 \%)$ to $0 / 30(0 \%)$ ( $p<0.05)$. Unnecessary antibiotic exposure rate was $7.7 \%$ in the PRE group versus $0.0 \%$ in the POST group $(\mathrm{p}<0.05)$.

Conclusion Implementation of this collection bundle reduced the number of false positive blood culture results. This has a potential benefit in reducing unnecessary antibiotic use and associated health care costs.

\section{PS-215 SEPSIS REDUCTION CARE BUNDLES IMPROVE COGNITIVE OUTCOME IN VERY LOW BIRTH WEIGHT INFANTS}

${ }^{1} \mathrm{JW}$ Davis, ${ }^{2} \mathrm{~S} J a r y,{ }^{1} \mathrm{PA}$ Cairns, ${ }^{1} \mathrm{D}$ Harding, ${ }^{2} \mathrm{~K}$ Luyt. ${ }^{1}$ Neonatology, St. Michael's Hospital University Hopsitals Bristol, Bristol, UK; ${ }^{2}$ Neonatal Neuroscience, University of Bristol, Bristol, UK
Background Very low birth weight (VLBW) infants with late onset sepsis have increased risk of neurodisability. Care bundles to reduce these infections in NICU are effective. The impact of care bundles on long-term neurodevelopmental outcome has not been described. We aimed to determine if implementation of a sepsis-reduction care bundle was associated with improvement in neurodevelopmental outcomes in VLBW infants.

Methods A multimodal sepsis improvement bundle was implemented in a regional NICU from July 2006. This bundle focused on hand hygiene and line care improvements. Mortality and neurological morbidity rates were compared pre- and post intervention (Jan '01 - Dec '07 vs. Jul '08 - Dec '12). Infants had neurodevelopmental assessment at 24 months corrected gestation with Bayley Scales of Infant development. Moderate cognitive disability was defined as a cognitive/language score below 2SDs, moderate motor disability as a motor score below 2SDs.

Results Birth weight, gestation and gender were similar in both cohorts. Coagulase Negative Staphylococcus septicaemia rates reduced from $7 / 1000$ care days before implementation to 2.8 / 1000 in 2012. Mortality rates were similar between the groups (66/426 vs. $40 / 310 ; p=0.3)$. There was no difference in moderate motor disability ( $17 / 85$ vs. $3 / 42 ; \mathrm{p}=0.07)$. There was a significant reduction in moderate cognitive disability (16/86 vs. $2 / 44 ; p=0.03$ ) after implementation of the sepsis care bundle.

Conclusions Sepsis-reduction care bundles improve the 2-year neurodevelopmental outcome of VLBW infants. The improvement seen in cognitive function is likely to translate into significantly less long-term learning disability.

\section{PS-216 MULTICENTRE PROSPECTIVE STUDY IN THE MANAGEMENT OF PRESUMED OR CONFIRMED EARLY ONSET SEPSIS AND INITIATION OF TREATMENT IN THE TERM AND PRETERM NEONATAL ADMISSIONS}

${ }^{1} \mathrm{M}$ Chalia, ${ }^{2} \mathrm{M}$ Upton. ${ }^{1}$ Neonatal UnitCambridge University Hospitals NHS Foundation Trust Addenbrookes Hospital, Cambridge, UK; ${ }^{2}$ Neonatal Network, East of England Deanery, Cambridge, UK

\subsection{6/archdischild-2014-307384.515}

Aim To assess the choice of antibiotics in correlation to clinical management of suspected or confirmed early onset neonatal sepsis. To identify the requirement for updating our practice within the East of England Neonatal Network.

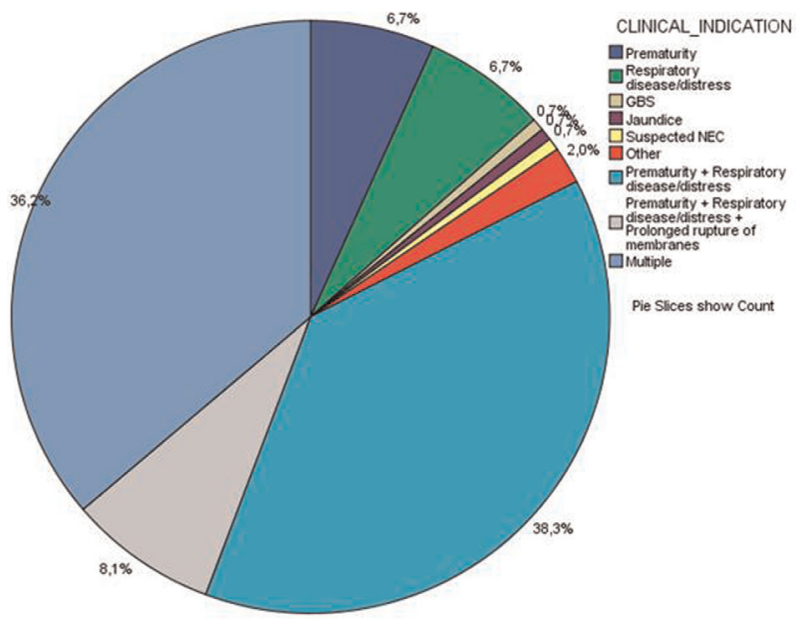

Abstract PS-216 Figure 1 


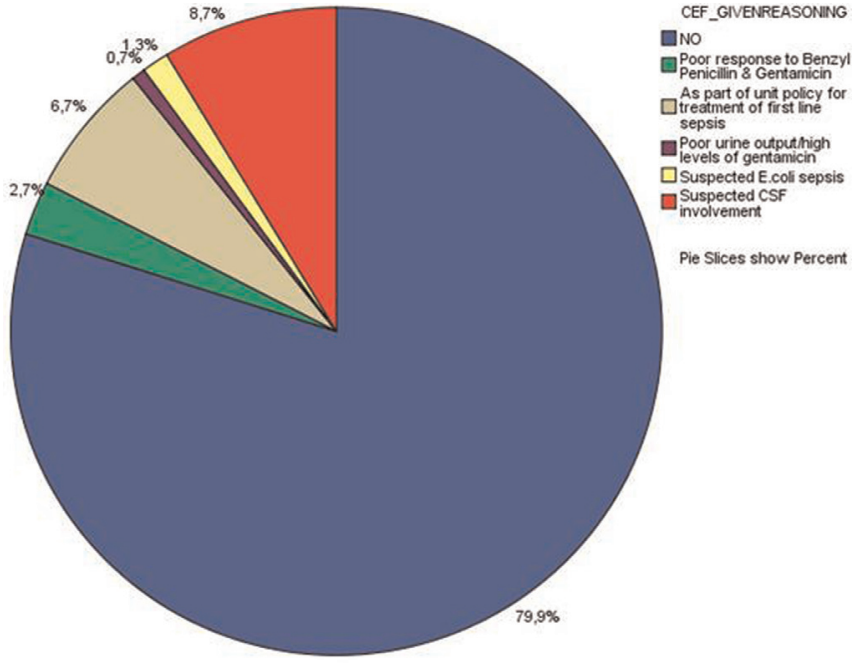

Abstract PS-216 Figure 2

Background Clinical practice within the network is facilitated by a regional guideline and a relevant clinical guidance tool from the National Institute for Health and Clinical Excellence (NICE149, August 2012).

Methods Prospective study from September 2012 until May 2013. 17 participating neonatal units within the network. 10 cases from each unit were included. These referred to term or preterm infants who required intravenous antibiotics for suspected or confirmed early onset sepsis with a minimum length of stay of 10 days. The data were analysed using SPSS 17.0.

Results 15 units participated. 149 babies were recruited with a mean gestational age of $32+2$ weeks. $91.3 \%$ of babies received intravenous benzyl penicillin and gentamicin as first line treatment. In $25 \%$ of cases there were prescribing issues regarding gentamicin. $20.1 \%$ received cefotaxime. $19.5 \%$ of babies underwent a lumbar puncture. $17.5 \%$ of babies received antifungal agents. In $15.4 \%$ of which as treatment.

Conclusions The overall outcome was positive with prompt recognition of risk factors and initiation of treatment across all units. This unified policy promotes good quality of care. However, the percentage of prescribing issues regarding gentamicin was worryingly high. Hence, further studies and review of literature are required to evaluate the efficiency of our practice and to establish alternative choice of antibiotics.

\section{PS-217 IMPACT OF THE NEW ALGORITHM FOR MANAGEMENT OF NEWBORNS WITH RESPECT TO RISK FOR EARLY-ONSET GBS DISEASE}

M García Díaz, T González Martínez, V García González, J GonzáLez García, B Fernández Martinez, C Pérez Mendez. Servicio de Pediatria, Hospital de Cabueñes, Gijon, Spain

\subsection{6/archdischild-2014-307384.516}

Background Guidelines for the prevention of perinatal group B streptococcal (GBS) disease were updated in 2010, including a revised algorithm for management of newborns with respect to risk for early-onset GBS disease (EOD-GBS).

Aim To know the impact of this new algorithm on EOD-GBS evaluations, hospital admissions, and detection of EOD-GBS cases in a newborn unit.

Methods Retrospective cohort study of infants of GBS-colonised mothers born at $\geq 36$ weeks gestational age in two periods of time: from July to December 2010, and from July to December
2012. The following variables were analysed: gender, gestational age, chorioamnionitis, indication for and prescription of antibiotics to the mother, EOD-GBS evaluations, infant admission and outcome. Continuous data were compared by using $t$ test; discrete data using chi square. Preventable fraction in the exposed $\left(\mathrm{Pf}_{\mathrm{e}}\right)$ was used to quantify the impact or the new algorithm.

Results One hundred and fifty-two neonates were included in 2010 and 130 in 2012. No significant differences between both groups were found regarding gender, gestational age, chorioamnionitis, obstetric care and antibiotic prophylaxis received by mothers. There were no cases of GBS infection in both periods. The new algorithm avoided $88 \%$ evaluations in EOD-GBS screening from 2010 to $2012\left(\mathrm{Pf}_{\mathrm{e}}=0.88,95 \%\right.$ confidence interval [CI]: 0.39-0.96). The number of infants admitted for suspected EOD-GBS was reduced by $48.1 \%\left(\mathrm{Pf}_{\mathrm{e}}: 0.481,95 \% \mathrm{CI}:-0.648-0.864\right)$. Conclusions Implementation of the 2010 algorithm resulted in a decrease of EOD-GBS evaluations and the number of newborn admissions for suspected sepsis.

\section{PS-218 DEVELOPING A SAFER DOSING REGIMEN OF AMIKACIN IN PRETERM INFANTS}

${ }^{1}$ SS Pereira, ${ }^{2}$ ST Kempley, ${ }^{1}$ AK Sinha. ${ }^{1}$ Department of Neonatology, Royal London Hospital, London, UK; ${ }^{2}$ Department of Neonatology, Barts and the London School of Medicine and Dentistry Queen Mary University of London, London, UK

\subsection{6/archdischild-2014-307384.517}

Background and aims Aminoglycosides with penicillin are commonly used first line antimicrobials of choice to target early onset sepsis organisms (1). The concern about emergence of gentamicin resistant gram-negative organism has led neonatal units to switch to Amikacin (2). The current British National formulary $(\mathrm{BNFc})$ recommends a dose of $15 \mathrm{mg} / \mathrm{kg} /$ day even for preterm infants. The ideal dose of amikacin for use in preterm infants is not clearly defined. The aim of this study was to compare amikacin blood levels in preterm infants less than 32 weeks gestation receiving two dosage regimens.

Methods During initial six-month period infants received amikacin dose of $12 \mathrm{mg} / \mathrm{kg} /$ day (group 1) and subsequently after a change to $12 \mathrm{mg} / \mathrm{kg}$ every $36 \mathrm{~h}$ (group 2) at the neonatal unit over a four-month period at Royal London Hospital. Data was collected from the neonatal database, hospital records and drug chart. Study was approved by Clinical effectiveness unit and Chi-square tests used for analysis (SPSS v22).

Results

Conclusions Most preterm infants even on a lower dose of amikacin than currently recommended by BNFc develop a high

\section{Abstract PS-218 Table 1}

\begin{tabular}{|c|c|c|}
\hline & $\begin{array}{c}\text { Group 1(amikacin } \\
12 \mathrm{mg} / \mathrm{kg} / \mathrm{day})\end{array}$ & $\begin{array}{c}\text { Group } 2 \text { (amikacin } \\
12 \mathrm{mg} / \mathrm{kg} 36 \\
\text { hourly) }\end{array}$ \\
\hline $\mathrm{N}$ & 46 & 18 \\
\hline No of babies with high trough amikacin levels (\%) & $35(76)^{\star}$ & $6(33)^{\star}$ \\
\hline Mean gestation in weeks (SD) & $28.3(2.8)$ & $28.0(2.7)$ \\
\hline Mean Birth weight in grams (SD) & $1129(354)$ & $1020(360)$ \\
\hline Mean Creatinine (range) in micromol/L & $78(36-111)$ & $79(20-140)$ \\
\hline
\end{tabular}

